\title{
Features of biochemical parameters of blood serum in patients with stable angina and type 2 diabetes mellitus
}

\begin{abstract}
Background: The study of a wide panel of vascular inflammatory markers is required to clarify the nature of the relationship between the pathogenetic mechanisms of coronary artery disease (CAD) and type 2 diabetes mellitus.
\end{abstract}

Aim: To conduct comparative analysis of lipid profile and markers of vascular inflammatory reaction in patients with $\mathrm{CAD}$, stable angina, without and together with DM 2 type.

Materials and methods: 169patients (males and females) aged 61.4 \pm 9.2 years with CAD and stable effort angina were studied. Patients were divided into two groups: group $1(n=123)$ with CAD, group 2 CAD patients with DM 2 type $(n=46)$. Study of blood plasma laboratory parameters was conducted in the groups of patients initially at the admission before coronary angiography.

Results: In both groups of patients, the excess of the reference values of the lipid profile atherogenic parameters (TC, LDL, VLDL, TG) was detected. Significant excess of the level of vascular inflammation markers (hs-CRP, TNF $-\alpha$, homocysteine, IL-1 $\beta$ ) was registered along with the excess of normal values of MMP-9 and endothelin-1 in the group of patients with CAD with DM type 2 that could indicate more pronounced vascular inflammation in this group.

Conclusion: Characteristic features for patients with CAD combined with DM type 2 are dyslipidemia with elevated total cholesterol (TC) level and low level of highdensity lipoprotein cholesterol (HDL), along with hyperactivation of markers of vascular inflammatory response with a significantly higher level of hs-CRP, TNF- $\alpha$, homocysteine and IL-1 $\beta$ compared to CAD patients without type $2 \mathrm{DM}$.

Keywords: diabetes mellitus type 2, coronary artery disease, lipid profile, inflammatory markers

\author{
Volume 6 Issue I - 2018
}

\section{Tatiana I Petelina, Natalia A Musikhina, Liudmila I Gapon, Ylia A Sharoyn, Elena A Gorbatenko}

Tyumen Cardiology Research Center,Tomsk National Research Medical Center, Russian Academy of Science, Russia

Correspondence: Petelina Tatyana Ivanovna, Senior Research Associate, Academy of Science, Tyumen Cardiology Research Center,Tomsk National Research Medical Center,Tomsk, 625026, Russia, Email petelina@cardio.tmn.ru

Received: December 22, 2017 | Published: January 09, 2018

\section{Introduction}

The incidence of diabetes mellitus (DM) doubles every 10-15years, acquiring the character of a non-infectious epidemic. At the same time, the real rates of increase in morbidity far outstrip even the most depressing predictions of statisticians. According to the latest data, the number of patients with diabetes in the world by the end of 2014 reached 387million people. According to the State Register of patients with diabetes, as of January 1, 2015, there were 4.1million cases registered in Russia, of which the number of patients with type 2 diabetes was 3.7 million. ${ }^{1}$ By 2030 , the number of patients with diabetes is expected to increase to 438.7 million, which is $7.7 \%$ of the adult population. ${ }^{2,3} \mathrm{SD}$ is a powerful factor in the development and progression of cardiovascular pathology. Development of diabetes increases the risk of cardiovascular mortality by 2 times in men and 4 times in women, which determines these patients in the category of high and very high cardiovascular risk. ${ }^{4}$

Despite intensive studies of atherosclerosis associated with DM, the mechanisms of the close relationship between diabetes and CAD are not yet fully indicated; as a result, the search for other causes that accelerate and aggravate the development of atherosclerotic vascular lesions and complications when combined with clinical conditions has been extensively conducted. ${ }^{5,6}$ Nowadays there is no doubt that in the pathogenesis of vascular lesions in atherosclerosis the important role play factors of inflammation and procoagulants circulating in blood. The role of chronic inflammatory process is under active discussion. ${ }^{7-10}$

\section{Aim}

To conduct comparative analysis of lipid profile and markers of vascular inflammatory reaction in patients with $\mathrm{CAD}$, stable angina, without and together with DM 2type.

\section{Materials and methods}

169patients (males and females) aged 61.4 \pm 9.2 years with CAD, stable effort angina and significant coronary artery stenosis were studied. Patients were randomized into two groups: group $1(n=123)$ without DM 2 type, group $2(n=46)$ with DM 2 type. Study of blood plasma laboratory parameters was conducted in the groups of patients initially at the admission stage before coronary angiography. Study protocol is approved by Ethic committee of the institute. Prior to be included into the study each of the participants provided written informative approval on using study results for the research needs. 
The study was performed in conditions of real clinical practice during standard therapy of CAD patients which included ACE inhibitors, $\beta$ - adrenergic blocking agents, calcium antagonists, disaggregants, statins, and nitrates for both groups and oral antidiabetic drugs for the patients from group 2. At the pre-admission stage no significant therapy differences between the groups were revealed.

Exclusion criteria were the following: acute coronary syndrome aged less than 30days, acute cerebral blood flow disorder aged less than 6months, DM 1 type, congestive heart failure (CAD) of functional class (FC) IV (as per NYHA), oncological and psychiatric diseases. Diagnostics of CAD forms and comorbidities was performed according to the acting ESC, Society of cardiology of Russian Federation and EASD recommendations.

Lipid profile parameters (total cholesterol (TC), triglycerides, HDL, LDL cholesterol, VLDL cholesterol, lipoprotein (a), Apo-A, Apo-B ( Cobas Integra 400 plus»); inflammatory markers (hs-CRP, TNFalpha, homocysteine, interleukine $1 \beta, 6,8$ («IMMULITE 1000»), sCD40 L, MMP-9, TIMP-1(«Bender MedSystems and Bioscience company»); endothelial dysfunction markers (endothelin-1, nitrites («Personal Lab» and «Dynatech») were measured.

Statistical data processing was conducted using application software package Statistica (SPSS Inc, ver 11.5). Comparison of the groups was done using Wilcoxon test for paired measurements. The data was presented as $\mathrm{M} \pm \mathrm{SD}$ - «average value \pm standard deviation», $\mathrm{p}<0.05$. Estimation of association between characteristics subject to normal and abnormal distributions was carried out using the Pearson and Spearman rank correlation coefficients, respectively.

\section{Results and discussion}

Patients of group 1 and 2 included in the study did not have significant differences $(p>0.05)$ in age, gender, CAD history, earlier myocardial infarction in past medical history, having comorbidities such as arterial hypertension, dyslipidemia, smoking. The majority of patients in both groups had effort angina II FC (64.1\% and 51.5\%) and obesity I class $(53.1 \%$ and $42.4 \%)$, respectively. Biochemical values in general groups of patients with CAD with and without DM 2 type are given in the Table 1. Estimation of lipid profile showed significant excess of TC $(\mathrm{p}=0.028)$ in the group with DM 2 type. Besides, excess of reference values of such atherogenic parameters as LDL, TG, VLDL in both groups of patients and significant excess of Lp (a) $(\mathrm{p}=0.015)$ in group 1 were noticed.

It is well known that DM 2 type having special quantitative changes also has important qualitative changes in lipid profile, which additionally enhanced its atherogenic potential. In the result of nonenzymatic glycosylation of apoproteins disorder of LDL clearance and collecting of small solid elements of this class is happening. These changes of lipoproteins structure are considered as one of the most important reasons of accelerated development of atherosclerosis with DM 2 type. ${ }^{11}$

Hypertriglyceridemia (HTG) is a more rigid marker of CAD in patients with DM 2 type than in people without hyperglycemia. As per the data of 11 years prospective DM patients monitoring (Paris Prospective Study), level of blood TG was connected with death risk of CAD. According to opinion of M. Laakso and co-authors important prognostic meaning of disease and death of $\mathrm{CAD}$ and its complications in patients with DM 2 type lies in lowering of HDL, decrease of which less than $0.9 \mathrm{mmol} / 1$ is accompanied by 4 times increase of death risk of cardiac pathology. ${ }^{11}$ According to our study trend of lowering of HDL with trend to increase of TG, LDL and VLDL is revealed in group of patients with DM 2 type.

Having direct correlational interrelations of average power $(\mathrm{r}=0.4-0.5)$ between glicated hemoglobin and values of atherogenic dyslipidemia: TC $(\mathrm{p}=0.02)$, VLDL $(\mathrm{p}=0.04)$, Apo-B $(\mathrm{p}=0.01)$ and TG $(p=0.003)$ among patients with DM 2 type proves that patients of group 2 have diabetic dyslipidemia. Diabetic dyslipidemia relates to the risk of CAD as well as isolated mild hypercholesterolemia. ${ }^{12}$

Mean value of LP (a) in group 1 and 2 was on the border level $(30.51 \pm 27.05$ and $20.61 \pm 19.95 \mathrm{mg} / \mathrm{dl}$, respectively) but the fact of the possibility of cardiovascular accidents and due to structural similarity of $\mathrm{Lp}$ (a) with plasminogen allows to regard it as competitive antagonist of the last one and can be associated with excess risk of coronary thrombosis. According to retrospective studies exceeded levels of Lp (a) are connected with progress of atherosclerotic plaques in the coronary arteries, which never had stenosis before. ${ }^{13,14}$ It is considered that chronic subclinical inflammation is a part of insulin resistance syndrome and cytokines are predictors of vascular complications of DM 2 type. ${ }^{9,15,16}$

The second group of patients had considerable hyperactivation of markers of system and local inflammation responds: hs-CRP $(p=0.007)$, homocysteine $(p=0.001), T N F-\alpha \quad(p=0.051)$. Levels of IL-6 and IL-8 had a tendency to values rise, within standard values, in comparison with group 1.

Besides, group with DM 2 type has significant excess of cytokine IL-1 $\beta(p=0.002)$ which is the main mediator responsible for local inflammatory response and acute phase response of the organism. According to the literature coronary blood flow disorder and myocardial ischemia leads to increase of its content in blood. Also, there is information about no system activation of Il-1 $\beta$ in case of stable and unstable angina. There is opinion that initiation of smoldering inflammation connected with rise of basic hs-CRP leads to insulin resistance and inductors of inflammation are proinflammatory cytokins, specially interleukins IL-6 and IL- $1 \beta .{ }^{16-18}$

Today increase of homocysteine in blood plasma is considered as significant risk factor of atherosclerotic vascular disease. It is supposed that homocysteine is subjected to autoxidation forming free radicals which damage vascular endothelium with further endothelium dysfunction. It starts a complicated set of enzymatic reactions leading to induction of TC synthesis and LDL oxidation, which stimulates atherogenic processes. ${ }^{19,20}$ More than 80 clinical and epidemiological studies have been performed and it was confirmed that HTG is one of the most important and independent risk factors of early and quick progress of atherosclerosis and coronary thrombosis. Clinical studies revealed that due to the oxidation stress HTG encourages insulin resistance and beta cells dysfunction accelerating DM progress. ${ }^{21}$ Besides, Fonseca $\mathrm{V}$ et al. in his rat study showed that increase of homocysteine concentration in blood may appear with hyperinsulinemia in the result of insulin injection; it may cause the vicious circle in case of insulin dependent DM.

In groups 1 and 2 elevated but significantly not different values of local inflammatory reactions -MMP-9 and lowering of TIMP-1 were revealed; it corresponds results of experimental and clinical studies showing tendency to growth of proinflammatory cytokines and proteinas with decrease of activity of anti-inflammatory mediators in case of DM 2 type. 
Table I Biochemical values in general groups of patients with CAD with and without DM 2 type (M $\pm S D)$

\begin{tabular}{|c|c|c|c|}
\hline Values & $\begin{array}{l}\text { CAD Patients without DM } 2 \\
\text { Type }(n=\mid 23)\end{array}$ & CAD Patients with DM $(n=46)$ & $P$ \\
\hline \multicolumn{4}{|l|}{ Lipid profile } \\
\hline TC (mmol/l) & $4.93 \pm 1.226$ & $5.43 \pm 1.12$ & 0.028 \\
\hline $\mathrm{HDL}(\mathrm{mmol} / \mathrm{l})$ & $1.12 \pm 0.26$ & $1.10 \pm 0.22$ & 0.080 \\
\hline LDL (mmol/l) & $2.91 \pm 1.01$ & $3.01 \pm 0.80$ & 0.603 \\
\hline $\mathrm{TG}(\mathrm{mmol} / \mathrm{l})$ & $2.02 \pm 0.83$ & $2.23 \pm 1.15$ & 0.549 \\
\hline VLDL (mmol/l) & $0.76 \pm 0.29$ & $0.83 \pm 0.315$ & 0.215 \\
\hline Lp-(a) (mg/dl) & $30.5 I \pm 27.05$ & $20.61 \pm 19.95$ & 0.015 \\
\hline Apo -AI (mg/dl) & $|49.0| \pm 24.85$ & $153.42 \pm 27.16$ & 0.322 \\
\hline Ap-B (mg/dl) & $86.52 \pm 21.89$ & $91.73 \pm 22.17$ & 0.173 \\
\hline \multicolumn{4}{|l|}{ Inflammatory markers } \\
\hline hs-CRP (mg/l) & $3.01 \pm 1.19$ & $3.52 \pm 1.21$ & 0.007 \\
\hline TNF- $\alpha(\mathrm{pg} / \mathrm{ml})$ & $9.8 I \pm 3.2 I$ & $10.83 \pm 3.61$ & 0.051 \\
\hline Homocysteine (mcmol/l) & $|2.34 \pm 5.6|$ & $15.73 \pm 6.33$ & 0.001 \\
\hline IL-Iv (pg/ml) & $4.4 I \pm I .27$ & $5.25 \pm 1.65$ & 0.002 \\
\hline IL -6 (pg/ml) & $3.73 \pm 2.58$ & $4.34 \pm 3.37$ & 0.357 \\
\hline IL -8 (pg/ml) & $16.22 \pm 12.96$ & $19.84 \pm 16.37$ & 0.166 \\
\hline CD 40 (ng/ml) & $94.44 \pm 36.18$ & $92.05 \pm 40.85$ & 0.623 \\
\hline sCD40L (ng/ml) & $3.5 I \pm 1.01$ & $3.54 \pm 1.16$ & 0.873 \\
\hline TIMP - I (ng/ml) & $90.22 \pm 17.24$ & $90.16 \pm 15.09$ & 0.967 \\
\hline MMP-9 (ng/ml) & $95.93 \pm 31.02$ & $92.64 \pm 31.10$ & 0.878 \\
\hline \multicolumn{4}{|l|}{ Endothelial dysfunction } \\
\hline Endotheline -I (fmol/I) & $\mathrm{I} .02 \pm 0.4 \mathrm{I}$ & $0.90 \pm 0.477$ & 0.114 \\
\hline Nitrites (nmol/l) & $3.02 \pm 1.24$ & $2.94 \pm 1.30$ & 0.599 \\
\hline \multicolumn{4}{|c|}{ Carbohydrate metabolism } \\
\hline Blood glucose (mmol/l) & $5.38 \pm 0.79$ & $7.65 \pm 1.98$ & 0.000 \\
\hline Glicated hemoglobin (\%) & $5.5 \pm 0.50$ & $7.1 \pm 1.00$ & 0.000 \\
\hline
\end{tabular}

Note: $n$ :Amount of patients; p: Significance of differences.

Among signal molecules participating in immune reactions and inflammation, an important role belongs to the system «receptor CD 40 - ligand CD40». Their expression is found in lymphocytes, monocytes, thrombocytes, endothelial cells, $\beta$ cells of insula of pancreatic gland, adipocytes and other tissues. System «receptor CD 40-L CD40» participates in forming if immune inflammatory responses in cardiovascular system and thrombosis. Signals realized through receptor CD 40 are involved in atherosclerosis and diabetic nephropathy; this allowed regarding this system as a universal element of pathogenesis uniting inflammatory disorders, hyperglycemia and vascular complications DM. ${ }^{10}$ Among indicators of signal inflammatory system (CD 40, sCD40L) our study did not find any significant differences and elevated values in both groups; it could possibly be connected with lack of patients.

Besides, it should be noted that prognostic importance of the values used today for estimation of endothelial function is not entirely studied. It is supposed that in case of DM 2type hyperhomocysteinemia can confound endothelial dysfunction, accelerate atherosclerotic processes, raise oxidative stress, lower thromboresistance, raise aggregative platelets ability and their adhesive features. Experimental and clinical studies showed that patients with DM 2 type had endothelial dysfunction. Hyperglycemia activates protein kinase $\mathrm{C}$ in endothelial cells, it may cause production of vasoconstrictive prostaglandins, endothelin-1 and angiotensine transforming enzyme which have direct or indirect harmful impact on vasomotor response..$^{20}$ Our study did not reveal any significant difference in endothelium 1-21 and nitrites in the main groups of patients, however, value of endothelium-1 was higher and nitrites value was lower than reference values in both groups of patients.

\section{Conclusion}

Results of a comparative analysis of biochemical parameters indicate that along with practically equally increased levels of atherogenic parameters of lipids profile and disorganization of 
endothelial system in patients with CAD, stable angina and diabetes mellitus type 2, compared to patients with CAD but without diabetes mellitus, significant hyperactivation of markers of vascular inflammatory response due to highly sensitive C-reactive protein, homocysteine and interleukin-1 was detected. The degree of systemic inflammation associated with chronic hyperglycemia in patients with $\mathrm{CAD}$ and diabetes mellitus can be considered as the most important characteristic indicating a serious risk for cardiovascular complications including destabilizing changes within the atherosclerotic plaque and atherothrombosis.

\section{Additional information}

\section{Source of financing}

Within the research program of Tyumen Cardiology Research Center, Tomsk National Research Medical Center, Russian Academy of Sciences, Tomsk, Russia.

\section{Acknowledgments}

None.

\section{Conflicts of interest}

Authors have no obvious and potential conflicts of interest to declare concerning the publishing of this article.

\section{Author's participation}

Tatiana I. Petelina: concept, work design, analysis of results, writing the article; Natalia A. Musikhina: analysis of results, writing the article; Liudmila I. Gapon: analysis of results, writing the article; Ylia A. Sharoyn: analysis of results; Elena A. Gorbatenko: statistical processing and analysis of the collected data.

\section{References}

1. Golukhova EZ, Kuznetsova EV. Myocardial revascularization in patients with coronary heart disease combined with type 2 diabetes mellitus:a review of modern technologies. Diabetes mellitus. 2016;19(5):406-413.

2. Volkov VI, Serik SA. Diabetes mellitus and coronary atherosclerosis. Atherosclerosis. 2011;7(2):16-22.

3. Shaw JE, Sicree RA, Zimmet PZ. Global estimates of the prevalence of diabetes for 2010-2030. Diabetes Res Clin Pract. 2010;87(1):4-14.

4. Dedov II, Shestakova MV, Galstyan GR. Standards of specialized diabetes care. In: Dedov II \& Shestakova MV, editors, $7^{\text {th }}$ edn, Diabetes Mellitus. 2015;18(1S):1-112.

5. Sumin AN, Bezdenezhnykh NA, Bezdenezhnykh AV. Effect of diabetes of the second type on the prevalence of multifocal atherosclerosis in patients with coronary heart disease. Cardiology. 2012;11:33-41.
6. Muir RL. Peripheral arterial disease: Pathophysiology, risk factors, diagnosis, treatment, and revention. $J$ Vasc Nurs. 2009;27(2):26-30.

7. Bezborodova TA, Tarasov AA, Reznikov EA. Diagnostic use of new pathogenic markers of vascular lesions in diabetic patients. Cytokines and inflammation. 2014;13(1):28-33.

8. Titov VN. The generality of atherosclerosis and inflammation:the specificity of atherosclerosis as an inflammatory process. Russian Medical Journa. 1999;5:44-49.

9. Gruzdev OV, Barbarash OL, Akbasheva OE. Markers of insulin resistance and inflammation in acute myocardial infarction. Cytokines and Inflammation. 2012;2:44-50.

10. Shevchenko OP, Prirodova OF, Orlova OV. CD 40 ligand in patients with coronary heart disease combined with type 2 diabetes. Cardiovascular therapy and prevention. 2006;5(7):101-111.

11. Janashia PH, Mirina EY. Violation of lipid metabolism in type 2 diabetes and its correction options. Russian Medical Journal. 2008;11:1561.

12. American Diabetes Association. Position Statement: Management of Dyslipidemia in Adults with Diabetes. Diabetes Care. 2003;26(Suppl 1):S83-S86.

13. Discepolo W, Wun T, Berglund L. Lipoprotein (a) and thrombocytes:potential mechanisms underlying cardiovascular risk. Pathophysiology of Haemostasis and Thrombosis. 2006;35(3-4):314-321.

14. Hartmann M, von Birgelen C, Mintz GS, et al. Relation between lipoprotein (a) and fibrinogen and serial intravascular ultrasound plaque progression in left main coronary arteries. $J$ Am Coll Cardiol. 2006;48(3):446-452.

15. Dehghan A, Kardys I, de Maat MP, et al. Genetic variation, C-reactive protein levels, and incidence of diabetes. Diabetes. 2007;56(3):872-878.

16. Gusev DE, Potievsky BG, Raychevin NA. Markers of inflammation in different forms of coronary heart disease. Cardiology. 2012; 4:4-8

17. Klimontov VV, Tyan NV, Fazullina ON, et al. Clinical and metabolic factors associated with chronic inflammation of low intensity in patients with diabetes mellitus typa 2. Diabetes mellitus. 2016;19(4):295-302.

18. Babayev AR, Tarasov AA, Bezborodov TA. The concept of systemic inflammation in the pathogenesis of diabetic angiopathy. Bulletin of Volgograd State Medical University. 2010;1(33):308.

19. Kravchuk NA. Hyperhomocysteinemia, cardiovascular disease and type 2 diabetes. Health Ukrainy. 2012;4:52-56.

20. Davidchek EV, Snezitckyi VA, Niconova LV. Relationship of hyperhomocysteinemia with coronary heart disease and Diabetes mellitus. Education Establishment Grodno State Medical University. 2015;1:9-13.

21. Potter K. The oxidant stress of hyperhomocysteinemia. Clin Biochem. 2008;45:27-30. 